\title{
UNIQUENESS THEOREM FOR THE REDUCED WAVE EQUATION UNDER AN NTH ORDER DIFFERENTIAL BOUNDARY CONDITION $^{1,2}$
}

\author{
R. C. MORGAN ${ }^{3}$
}

Introduction. In this paper, uriqueness is demonstrated for the solution, $u(x, y)$, to a mathematical problem that arises in the phenomenological theory of multi-mode surface wave propagation (see Karal and Karp [1] and [2]). The unusual aspect is that a boundary value problem is posed using a boundary condition of the form

$$
\prod_{m=1}^{N}\left(\frac{\partial}{\partial y}+\lambda_{m}\right) u=0, \quad y=0 .
$$

Previously, Kane [3] proved uniqueness for the same boundary as here with $N=2$, restricting the $\lambda_{m}$ so that they could not be real positive quantities. This requirement excludes the possibility of exciting physically interesting surface waves of the form $\exp \left[-\lambda_{m} y\right.$ $\left.+i\left(K^{2}+\lambda_{m}^{2}\right)^{1 / 2}|x|\right]$. Furthermore, the far field nature of $u(x, y)$ was required to satisfy the condition

$$
\underset{K r \rightarrow \infty}{u(r, \theta)} \cong \frac{e^{i K r}}{(K r)^{1 / 2}} f(\theta)+O\left(\frac{1}{(K r)^{3 / 2}}\right), \quad 0 \leqq \theta \leqq \pi,
$$

where $f(\theta)$ is a continuous function of $\theta$. This radiation condition is more explicit than the usual Sommerfeld condition, and, by its very nature, rules out surface waves as they are oscillating and nondecaying along the boundary.

It was found to be equally convenient to state and prove uniqueness for all $N$ and $\lambda_{m} \neq \pm i K$. The explicit far field behavior mentioned above is not required; rather we assume a Sommerfeld condition. Hence, where we overlap with Kane [3], the required radiation condition is improved and the theorem is extended for higher order boundary conditions. Otherwise, in the case where surface waves arise, the results are independent.

Presented to the Society, April 4, 1966; received by the editors November 11, 1965.

1 This research was supported by the Air Force Office of Scientific Research under Contract No. AF-AFOSR-537-64. Reproduction in whole or in part is permitted for any purpose of the U.S. Government.

${ }^{2}$ Based in part on the author's doctoral dissertation.

${ }^{3}$ Currently at Saint John's University, Jamaica, New York. 
Basically, the formulation is a modification of the extended form of Stoker and Peter's work [4] used by Morgan and Karp [5], in the case of single mode surface wave incidence on a right-angled wedge. Furthermore, the solution to the problem is derived in Morgan, Karal and Karp [6].

The following theorem is demonstrated:

Theorem I. $N$-Mode UNIQUeness. Let $u(x, y)$ be a solution of the following boundary value problem:

I.1. $u(x, y)$ has continuous second order derivatives everywhere except at the point $\left(0, y_{0}\right)$ and satisfies the inhomogeneous wave equation

$$
\frac{\partial^{2} u}{\partial x^{2}}+\frac{\partial^{2} u}{\partial y^{2}}+K^{2} u=-4 \pi \delta(x) \delta\left(y-y_{0}\right)
$$

in $y \geqq 0$ (see Figure 1). Here $K$ is real and $y_{0}>0$.

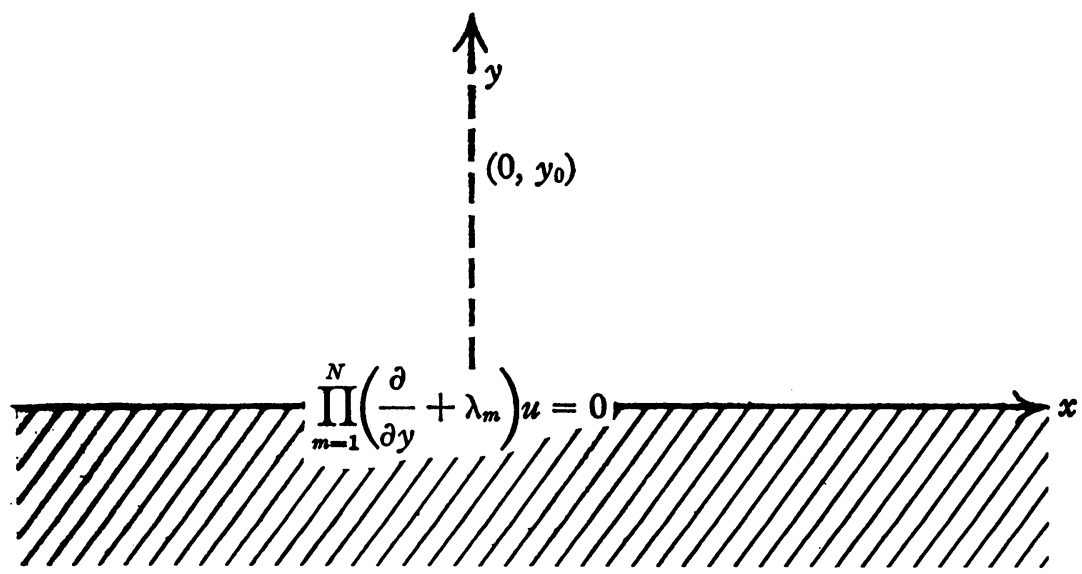

FIGURE 1

I.2. $\prod_{m=1}^{N}\left(\partial / \partial y+\lambda_{m}\right) u=0$ for $y=0$, where the $\lambda_{m}$ 's are distinct, nonvanishing and $\lambda_{m} \neq \pm i K$.

I.3. $u(x, y)$ can be written as

$$
u=\pi i H_{0}^{(1)}\left(K\left(x^{2}+\left(y-y_{0}\right)^{2}\right)^{1 / 2}\right)+w(x, y)
$$

where $w(x, y)$ has continuous second order derivatives everywhere and is a solution of the homogeneous wave equation. Furthermore, $w(x, y)$ may be decomposed as follows:

$$
w(x, y)=\sum_{m=1}^{N} C_{m} \exp \left[-\lambda_{m} y+i\left(K^{2}+\lambda_{m}^{2}\right)^{1 / 2}|x|\right]+u_{\mathrm{rad}}
$$


where the $C_{m}$ 's represent excitation coefficients.

I.4. In every region excluding the positive $y$-axis $u_{\mathrm{rad}}$ obeys the regularity condition

$$
\sum_{i=0}^{N+1} \sum_{j=0}^{i}\left|\frac{\partial^{i} u_{\mathrm{rad}}}{\partial x^{j} \partial y^{i-j}}\right| \leqq M(N)
$$

for $r>R_{0}$, where $M$ is independent of $r$ and $\theta$ while $R_{0}$ is some positive constant.

Furthermore, we assume that $u_{\mathrm{rad}}$ satisfies the radiation condition

$$
\lim _{r \rightarrow \infty}(r)^{1 / 2}\left(\frac{\partial u_{\mathrm{rad}}}{\partial r}-i K u_{\mathrm{rad}}\right)=\mathrm{C}
$$

uniformly in $\theta, 0 \leqq \theta \leqq \pi$, and vanishes at infinity.

Then $u(x, y)$ is uniquely determined.

The proof is accomplished in the following manner: We introduce $\psi(x, y)$ defined as the difference of two solutions and then show that it is identically zero. These solutions are allowed to have completely dissimilar but radiating diffracted fields while the excitation coefficients are not prescribed in the formulation.

We immediately note that the resulting $\psi$ problem is not separable in $r$ and $\theta$ due to the boundary condition. Thus, by the procedure of [1], we introduce an auxiliary function $v(x, y)$ so constructed that it satisfies the reduced wave equation and simple homogeneous boundary conditions. Next we deduce certain properties about $v$ which coupled with Weinstein's technique [7] enables us to determine this function uniquely. In this way, we show that $v$ is identically zero. Then, on inverting the auxiliary operator, we obtain $\psi(x, y)$. Following this, by requiring the continuity of $\partial \psi / \partial x$ across the positive $y$ axis, we demonstrate that $\psi$ is identically zero.

1. Proof of Theorem I. Let us consider two functions $u^{(1)}$ and $u^{(2)}$ that satisfy the conditions of Theorem I as follows:

$$
\begin{aligned}
& u^{(1)}=\pi i H_{0}^{(1)}\left(K r_{-}\right)+w^{(1)}, \\
& u^{(2)}=\pi i H_{0}^{(1)}\left(K r_{-}\right)+w^{(2)}
\end{aligned}
$$

where

$$
w^{(j)}=\sum_{m=1}^{N} C_{m}^{(j)} \exp \left\lfloor-\lambda_{m} y+i\left(K^{2}+\lambda_{m}^{2}\right)^{1 / 2}|x|\right\rfloor+u_{\mathrm{rad}}^{(j)}
$$

for $j=1,2$ and $r_{-}=\left(x^{2}+\left(y-y_{0}\right)^{2}\right)^{1 / 2}$. These functions are taken to 
have possibly different excitation coefficients and possibly different but radiating diffracted fields. Forming the difference function

$$
\psi(x, y)=u^{(1)}-u^{(2)} \equiv w^{(1)}-w^{(2)},
$$

one easily sees that $\psi$ is a solution of the boundary value problem posed in Theorem I, except $M$ in Condition (I.4a) is replaced by $2 M$, and $\psi$ is without a source term. Consequently, ${ }^{4}$

$$
\psi(x, y)=\psi_{\mathrm{e} . \mathrm{m} .}+\psi_{\mathrm{rad}}
$$

where

$$
\psi_{\mathrm{e} . \mathrm{m} .}=\sum_{m=1}^{N}\left(C_{m}^{(1)}-C_{m}^{(2)}\right) \exp \left[-\lambda_{m} y+i\left(K^{2}+\lambda_{m}^{2}\right)^{1 / 2}|x|\right]
$$

and

$$
\psi_{\mathrm{rad}}=u_{\mathrm{rad}}^{(1)}-u_{\mathrm{rad}}^{(2)}
$$

obeys the radiation condition of Theorem I. We will prove that $\psi$ is iden tically zero.

Let us define

$$
v(x, y)=\prod_{m=1}^{N}\left(\frac{\partial}{\partial y}+\lambda_{m}\right) \psi(x, y) .
$$

Upon using the properties of $\psi$, we prove the following theorem:

THEOREM I.A.

I.A.1. $v$ has continuous second order derivatives and is a solution of

$$
\frac{\partial^{2} v}{\partial x^{2}}+\frac{\partial^{2} v}{\partial y^{2}}+K^{2} v=0 \quad \text { in } y \geqq 0 .
$$

I.A.2. $v=0$ for $y=0$.

I.A.3a. $v$ and its derivatives obey the following regularity conditions:

$$
|v|+\left|\frac{\partial v}{\partial y}\right|+\left|\frac{\partial v}{\partial x}\right| \leqq P \exp +\left[|\lambda|_{M}+\left|\left(K^{2}+\lambda^{2}\right)^{1 / 2}\right|_{M}\right]_{r}
$$

for $r>R_{0}$, where $P$ is independent of $r$ and $\theta$, while $R_{0}$ is as in Theorem I, some positive constant. Here we define

$$
|\lambda|_{M} \equiv \max _{m}\left|\lambda_{m}\right| \text { and }
$$

4 We introduce the term $\psi_{\text {e.m. }}$ (e.m. $=$ excited mode) in place of $\psi_{\text {surface wave }}$ to indicate that we are including terms where $\operatorname{Re} \lambda_{m}<0$ which are not strictly speaking surface waves. 


$$
\left|\left(K^{2}+\lambda^{2}\right)^{1 / 2}\right|_{M} \equiv \max _{m}\left|\left(K^{2}+\lambda_{m}^{2}\right)^{1 / 2}\right|
$$

I.A.3b. $v=O(1)$ as $r \rightarrow 0$.

I.A.4. $v$ may be written as

$$
v=\prod_{m=1}^{N}\left(\frac{\partial}{\partial y}+\lambda_{m}\right) \psi_{\mathrm{rad}}
$$

for $\theta \neq \pi / 2$.

I.A.5. $v$ satisfies the set of conditions

$$
\lim _{r \rightarrow \infty}(r)^{1 / 2} \int_{0}^{\pi}\left[\frac{\partial v}{\partial r}-i K v\right] \sin n \theta d \theta=0, \quad n=1,2, \cdots .
$$

Proof. Conditions I.A.1 and I.A.2 follow immediately from I.3 and I.2. Condition I.A.3a is shown by applying I.3 and I.4a after observing that

$$
\begin{aligned}
& \left|\exp \left[-\lambda_{m} y+i\left(K^{2}+\lambda_{m}^{2}\right)^{1 / 2}|x|\right]\right| \\
& \quad \leqq \exp \left[\left|\lambda_{m}\right| y+\left|\left(K^{2}+\lambda_{m}^{2}\right)^{1 / 2}\right||x|\right] .
\end{aligned}
$$

Note that (1.8) is easily bounded by

$$
\exp \left[|\lambda|_{M} y+\left|\left(K^{2}+\lambda^{2}\right)^{1 / 2}\right|_{M}|x|\right]
$$

for all $m$. Obviously, we can obtain better bounds in special cases of $\lambda_{m}$; however, the above bound will suffice for all cases. Condition I.A.3a follows from property I.A.1. We see that I.A.4 holds by noting that the operator on $\psi$ annihilates the excited mode terms, thus

$$
\begin{aligned}
v & =\prod_{m=1}^{N}\left(\frac{\partial}{\partial y}+\lambda_{m}\right) \psi \\
& =\prod_{m=1}^{N}\left(\frac{\partial}{\partial y}+\lambda_{m}\right) \psi_{\mathrm{e} . \mathrm{m} .}+\prod_{m=1}^{N}\left(\frac{\partial}{\partial y}+\lambda_{m}\right) \psi_{\mathrm{rad}} \\
& =0+\prod_{m=1}^{N}\left(\frac{\partial}{\partial y}+\lambda_{m}\right) \psi_{\mathrm{rad}}
\end{aligned}
$$

holds for $\theta \neq \pi / 2$. This result doesn't hold for $\theta=\pi / 2$ since $v$ is a wave function everywhere while $\partial \psi_{\mathrm{rad}} / \partial x$ is discontinuous across the line $\theta=\pi / 2$. Before proceeding to prove I.A.5, let us set

$$
r^{1 / 2}\left(\frac{\partial}{\partial r}-i K\right)()=L() \text {. }
$$


Then, I.A.5 takes on the form

$$
\lim _{r \rightarrow \infty} \int_{0}^{\pi} L(v) \sin n \theta d \theta=0
$$

$n=1,2, \cdots$ It is intuitively clear that any condition of this type will rest heavily on the behavior of $\psi_{\text {rad }}$. Thus, we will replace $v$ by its equivalent form given in I.A.4, wherever possible, and elsewhere adjust the remaining interval of integration so that its net effect is small.

Towards this end, we write

$$
\int_{0}^{\pi} L(v) \sin n \theta d \theta=\int_{0}^{\pi / 2-\eta}+\int_{\pi / 2-\eta}^{\pi / 2+\eta}+\int_{\pi / 2+\eta}^{\pi}(L(v) \sin n \theta) d \theta
$$

where

$$
\eta=\frac{1}{r} \exp -\left[|\lambda|_{M}+\left|\left(K^{2}+\lambda^{2}\right)^{1 / 2}\right|_{M}\right] r
$$

Then on estimating the above in tegrals for large $r$ we will determine a function, $R\left(\epsilon_{n}\right)$ depending on $\epsilon_{n}, N$, and $M$ such that for any $\epsilon_{n}>0$ we have

$$
\left|\int_{0}^{\pi} L(v) \sin n \theta d \theta\right|<\epsilon_{n}
$$

for all $r>R\left(\epsilon_{n}\right)$.

A similar procedure was used in [5]. However, there $\eta$ was taken to be $1 / r$. Here it is necessary to include the exponential in the definition of $\eta$ in order to annihilate the possibly large behavior of excited modes having $\operatorname{Re} \lambda_{m}<0$. The integral over the interval $[\pi / 2-\eta$, $\pi / 2+\eta]$ will be small as a direct consequence of the smallness of the interval. The remaining integrals will be small because of $\psi_{\mathrm{rad}}$.

The proof proceeds as follows:

REMARK 1. From I.A.3a it follows on writing

$$
\frac{\partial v}{\partial r}=\frac{\partial v}{\partial x} \cos \theta+\frac{\partial v}{\partial y} \sin \theta
$$

that

$$
\left|\frac{\partial v}{\partial r}\right| \leqq P \exp +\left[|\lambda|_{M}+\left|\left(K^{2}+\lambda^{2}\right)^{1 / 2}\right|_{M}\right] r \text { for } r>R_{0} .
$$


Thus, on applying the usual maximum estimate for $r>R_{0}$, we have the bound

$$
\left|\int_{\pi / 2-\eta}^{\pi / 2+\eta} L(v) \sin n \theta d \theta\right| \leqq \frac{2[1+K] P}{r^{1 / 2}} .
$$

Note that here we made use of the special form of $\eta$ to cancel out the exponential term occurring in the estimate of $L(v)$. Using the pseudoradiation properties of radiating functions derived in Morgan [8], it is easy to estimate the remaining two integrals. Thus, one can now verify I.A.5.

2. We show, following the method of Weinstein [7], that the function $v \equiv 0$ is the unique solution for $v$. This is accomplished by expanding $v$ for fixed $r$ in the Fourier sine series

$$
v=\sum_{n=1}^{\infty} C_{n}(r) \sin n \theta
$$

and then demonstrating that $C_{n}(r) \equiv 0$ for all $n$.

First the properties of $v$ are used to show that the Fourier coefficients obey the Sommerfeld differential radiation condition (actually I.A.5) and are bounded at the origin. Consequently, from these considerations and the fact that $C_{n}(r)$ is given uniquely as

$$
C_{n}(r)=A_{n} H_{n}^{(1)}(K r)+B_{n} H_{n}^{(2)}(K r),
$$

it is easily seen that $C_{n}(r)$ is identically zero.

3. Lastly, on inverting the equation

$$
\prod_{m=1}^{N}\left(\frac{\partial}{\partial y}+\lambda_{m}\right) \psi=0
$$

and referring to (1.5), we arrive at the form

$$
\psi=\sum_{m=1}^{N}\left(C_{m}^{(1)}-C_{m}^{(2)}\right) \exp \left[-\lambda_{m} y+i\left(K^{2}+\lambda_{m}^{2}\right)^{1 / 2}|x|\right] .
$$

Then from the continuity of $\partial \psi / \partial x$ across the $y$-axis, one sees that $\psi$ must be taken identically zero. Hence, $u(x, y)$ is uniquely determined.

Acknowledgment. The author wishes to express his appreciation

5 The essential result pertinent to this proof is that the limit as $r \rightarrow \infty$ of the first and third integral in (1.13) is zero. 
to Professor S. N. Karp for his continued encouragement and guidance.

\section{BiBLIOGRAPHY}

1. F. C. Karal and S. N. Karp, Phenomenological theory of multi-mode surface wave excitation, propagation and diffraction. I, Plane Structures, New York Univ., Courant Inst. Math. Sci., Div. of Electromagnetic Res., Res. Rep. No. EM-198, 1964.

2. - - Phenomenological theory of multi-mode surface wave structures, QuasiOptics Symposium, Brooklyn Polytechnic Inst., Wiley, New York, 1964; New York Univ., Courant Inst. Math. Sci., Div. of Electromagnetic Res., Res. Rep. No. EM-201, 1964.

3. J. Kane, A uniqueness theorem for the reduced wave equation, Proc. Amer. Math. Soc. 12(1961), 967-972.

4. A. S. Peters and J. J. Stoker, A uniqueness theorem and a new solution for Sommerfeld's and other diffraction problems, Comm. Pure Appl. Math. 7 (1954), 565-585.

5. R. C. Morgan and S. N. Karp, Uniqueness theorem for a surface wave problem in electromagnetic diffraction theory, Comm. Pure Appl. Math. 16 (1963), 45-56; New York Univ., Courant Inst. Math. Sci., Div. of Electromagnetic Res., Res. Rep. No. EM-178, 1962.

6. R. C. Morgan, F. C. Karal and S. N. Karp, Solution to the phenomenological problem of a magnetic line source above a plane structure that supports $\mathrm{N}$-excited surface wave or leaky wave modes, New York Univ., Courant Inst. Math. Sci., Div. of Electromagnetic Res., Res. Rep. No. EM-215, 1965.

7. A. Weinstein, On surface waves, Canad. J. Math. 1 (1949), 271-278.

8. R. C. Morgan, Pseudo-radiation conditions for derivatives of radiating functions, New York Univ., Courant Inst. Math. Sci., Div. of Electromagnetic Res., Res. Rep. No. BR-54, 1965. [Cf. R. C. Morgan, Thesis; Chapter I, Part A.]

Courant Institute of Mathematical Sciences, New York University 